\title{
Carnets
}

Revue électronique d'études françaises de l'APEF

Deuxième série - 12 | 2018

Théorie Mimétique et Études Littéraires

\section{Rimbaud, Ramuz, Bosco : la forme poétique et ses vertus cathartiques}

\section{Michel Arouimi}

\section{OpenEdition}

Journals

\section{Édition électronique}

URL : http://journals.openedition.org/carnets/2457

DOI : $10.4000 /$ carnets. 2457

ISSN : 1646-7698

Éditeur

APEF

\section{Référence électronique}

Michel Arouimi, «Rimbaud, Ramuz, Bosco : la forme poétique et ses vertus cathartiques », Carnets [En ligne], Deuxième série - 12 | 2018, mis en ligne le 17 janvier 2018, consulté le 20 avril 2019. URL http://journals.openedition.org/carnets/2457 ; DOI : 10.4000/carnets.2457

Ce document a été généré automatiquement le 20 avril 2019.

\section{(c) (i) (8)}

Carnets est mis à disposition selon les termes de la licence Creative Commons - Atribution - Pas d'utilisation commerciale 4.0 International. 


\title{
Rimbaud, Ramuz, Bosco : la forme poétique et ses vertus cathartiques
}

\author{
Michel Arouimi
}

1 Rimbaud est connu pour la violence de sa poétique, étrangement liée à sa fascination pour le «Nombre et [...] l'Harmonie », mentionnés dans la plus fameuse de ses lettres comme le but de sa quête. Cette violence prend diverses formes, parmi lesquelles les conflits de doubles, dans certaines illuminations comme dans la section «Délires I » de Une saison en enfer, peuvent évoquer au lecteur moderne les aspects majeurs de la théorie de René Girard: la rivalité mimétique, engendrée par la contradiction émanant d'une instance paternelle. Cette analyse poétique de la violence est plus évidente chez C.-F. Ramuz, le poète et romancier suisse qui vouait une sorte de culte à Rimbaud. Ramuz a d'ailleurs été injustement considéré comme un écrivain "régionaliste»; son œuvre, comme celle de Rimbaud, est universelle, puisque les causes et les effets de la «violence fondatrice » selon Girard, se voient cernés dans les récits de Ramuz. Le rapprochement de ces écrivains peut s'étendre à Henri Bosco, poète et romancier français, dont la vision métaphysique est éclipsée elle aussi par son injuste réputation de " régionalisme »; Bosco est un autre poète universel, ne serait-ce que par les conflits de doubles, définis par luimême comme le thème majeur de son œuvre. Le mimétisme, mais encore et surtout les vertus cathartiques de la crise sacrificielle, tels que les cerne Girard, sont en effet questionnés par Bosco. L'association de Ramuz et de Bosco ne doit pas surprendre, et d'autant plus que le souvenir de Rimbaud hante maints romans de Bosco, même si ce dernier n'a pas avoué cette dépendance, sinon dans la citation de « Une Saison en Enfer » dans son premier roman publié.

Enfin ces trois auteurs ont en commun une vision singulière de la forme poétique, l'Harmonie chère à Rimbaud, qui n'en est pas moins l'objet d'une sorte de remise en cause implicite, dans les œuvres mêmes qui, chez Rimbaud et ses deux successeurs, répondent pourtant à ce critère. Les vertus cathartiques dont nous parlions sont-elles aussi celles de l'harmonie des formes artistiques? Ce problème qui sous-tend l'œuvre de ces trois auteurs n'est pas abordé dans la théorie de René Girard. Mais cette théorie n'en est pas 
moins anticipée sur le mode poétique par ces trois auteurs, aussi soucieux de leur art que d'un climat social délétère, dont cet art serait l'inefficace thérapie.

Dans Une saison en enfer, en particulier dans la section Délires II, sous-titrée « Alchimie du verbe »), Rimbaud fait le procès de son expérience poétique. Cette autocritique, non sans paradoxe, est en germe dans ses poèmes. Certains d'entre eux précisent d'ailleurs l'origine des tensions que cet art alchimique aurait pour fonction d'exorciser, dans la conciliation des valeurs opposées, sémantiques ou purement sonores. Les souvenirs familiaux qui ont en partie inspiré des poèmes comme Mémoire ou Comédie de la soif, sont dominés par l'image d'un pouvoir paternel ou parental défaillant. Au-delà de l'horizon biographique du poète, on peut y voir une représentation de la contradiction fondatrice, dont René Girard a indiqué le rôle inspirateur dans la culture humaine, sans insister sur les formes artistiques. Dans Délires I (premier volet du diptyque formant l'axe médian de Une saison en enfer), Rimbaud cerne justement les effets relationnels les plus répandus de cette contradiction. Le rapport de la Vierge folle et de l'époux infernal, dans Délires I, apparaît en effet comme une alchimie sentimentale ratée, un exemple vécu du mimétisme violent qui, d'après le lien de Délires I et Délires II, trouverait son antidote dans la pratique de l' «alchimie du verbe ». L'activité poétique, entendue par le "voyant» comme une ressaisie rénovatrice du monde extérieur, déplace d'ailleurs sur le terrain de l'art la dualité relationnelle qui est lot de tous les hommes - quand ce monde est un partenaire que défie le « voyant ».

4 Dans son poème Mémoire, qui compte parmi ses deniers poèmes en vers, Rimbaud évoque un cadre champêtre, peuplé de figures étranges qui réfèrent sans doute au vécu familial $\mathrm{du}$ jeune Arthur. Dans la dernière section du poème (qui compte cinq sections numérotées, chacune de deux strophes), la première strophe exprime le désarroi du locuteur : une vision florale qui cristallise les contradictions intimes de ce dernier, ou plutôt la contradiction émanant du Père selon Girard. En effet dans la section médiane, "le départ de l'homme " peut s'interpréter dans ce sens, même s'il s'agit du père charnel d'Arthur, éloigné de sa famille, mais obscurément présent dans l'esprit de ses enfants. (Sur un manuscrit récemment retrouvé du poème, la dédicace «D'Edgar Poe ", inscrite avant le titre qui fut d'abord Famille maudite, associe à cette thématique paternelle l'idée d'une filiation littéraire, vaguement concurrentielle). «Jouet de cet œil d'eau morne, Je n'y puis prendre, /oh canot immobile ! oh bras trop courts ! ni l'une/ ni l'autre fleur : ni la jaune qui m'importune, / là ; ni la bleue, amie à l'eau couleur de cendre » (Rimbaud, 2009 : 234-235). Ces images donnent en effet une forme esthétique à la contradiction dont l'origine est cernée dans la section médiane du poème, avec la séparation de "Madame " et de "l'homme»: "Lui, comme/ mille anges blancs qui se séparent sur la route». Sublime image, dont le sens angélique se corrompt par cette séparation démoniaque.

5 Cette image est encore celle de "l'inconnu » que le " voyant », dans la plus fameuse de ses lettres, se propose de débusquer au fond de lui-même, comme une vérité qui pourrait éclairer l'humanité. Si cette vérité a rapport au "Nombre et [à] l'Harmonie », que le voyant aspire à médiatiser dans ses poèmes, les violences physiques qui en sont le prix, exposées dans cette lettre, laissent perplexe. L'inconnu en question nommerait la contradiction violente, si volontiers représentée dans les poèmes, que les hommes ne dépassent que dans une contemplation ou célébration du « Nombre et de l'Harmonie ».

Dans la section médiane de Mémoire, les «mille anges blancs » suggèrent vaguement le rôle inspirateur de cette contradiction dans les formes connues du sacré. Les derniers mots du poème évoquent sur le mode symbolique les effets sociaux de cette 
contradiction, avec la chaine du canot tirée «à quelle boue? " La boue exprimant l'abolition des différences individuelles, fatale à la singularité de chaque être, dont ce canot, parmi d'autres motifs de cette section, est l'image. Les effets culturels les plus matériels de cette contradiction (la "violence de la monnaie », jadis étudiée par un disciple de René Girard) sont circonscrits dans la section 2 du poème, où l'idée de la valeur monétaire, dans un univers surchauffé, est associée aux motifs, juxtaposés dans le texte, du « miroir » et de la « jalousie».

On m'excusera de ne pas recreuser cette énigme, moins parlante que la position centrale du couple déjà souligné, qui s'impose comme la clef des dispositions symétriques du poème. Si ces effets de symétrie, que j'ai étudiés ailleurs, participent à l'harmonie de la construction du poème, ils n'en sont pas moins l'expression, et peut-être le remède, du déchirement de ce couple, intériorisé par le locuteur. Le paysage décrit est le cadre d'un autosacrifice, dont la raison est à chercher dans les tensions affrontées par les quelques figures humaines évoquées dans ce poème.

Il en va de même dans le poème Comédie de la soif, lui aussi de cinq sections, écrit la même année (1872). Dans la section 3, le désir suicidaire du locuteur qui préfère « Pourrir dans l'étang, / Sous cette affreuse crème", évoque la "boue " de Mémoire, avec des détails apocalyptiques que je ne peux souligner ici. Dans la section 4, l'égarement du poète alchimiste (ou du futur marchand ?) : « Si j'ai jamais quelque or/ Choisirai-je le Nord / Ou le pays des Vignes ?... » (Rimbaud, 2009 : 199-201), n'est pas moins expressif que la rivalité des deux fleurs hostiles de Mémoire. Mais dans la section 1 de cette Comédie (titre évocateur, qui exprime toutes les formes du mimétisme, inspirées par la contradiction qui nous occupe), l'évocation justement redoublée des «Grands-Parents", exprime le rôle d'une instance paternelle (au-delà de la biographie du poète) dans ce phénomène. Il s'agit d'ailleurs de défunts, apparemment revenus à la vie. Incarnation absolue de cette contradiction, ces personnages proposent à leur descendant des offrandes liquides, non moins parlantes: "Tiens, prends / Les liqueurs ...] / Le Thé, le Café, si rares ». La majuscule du Thé et du Café, boissons excitantes (les liqueurs ayant des vertus inverses) réinscrit la dualité dans cette énigme à laquelle concourent bien d'autres motifs.

9 Le vers le plus remarquable du poème se situe dans la section $2:$ «Divisez l'eau fine ». (Le sujet en est les " Ondines ", avec lesquelles contrastent savoureusement les "Juifs errants de Norwège » dans cette section). Ce vers divise lui-même en deux suites de vingt-six vers le dialogue, rapporté dans ce poème, du « Moi » avec différents partenaires. Une fois de plus la représentation poétique des effets de la double contrainte émanant du Père, accompagne des visées autocritiques intéressant la forme poétique. On peut comprendre ainsi le silence de Rimbaud, conséquence logique de cette découverte intuitive.

Ces poèmes sont l'objet de réminiscences variées dans les deux romans les plus apocalyptiques de C.-F. Ramuz, Les Signes parmi nous (1919) et Présence de la mort. (1922). Des réminiscences ou plutôt de géniales réinventions, sous la plume de ce poète romancier, soucieux de "vivre et de revivre Rimbaud» sans le "copier ». J'ai étudié ailleurs cette filiation (Arouimi, 2009), dominée en fait par les réminiscences du poème Enfance de Rimbaud, lui aussi de cinq sections! Dans la section I, les « terrasses voisines de la mer » où tournoient des Dames, sont empreintes d'un érotisme qui est absent de l'écho de ce motif au tout début de la section IV: «Je suis le saint, en prière sur la terrasse ». Des dames au saint, c'est toujours le drame d'un partage de l'être, obscurément lié aux drames sacrificiels qui sont l'objet d'une suite d'allusions dans la section médiane. 
11 Or, Ramuz, dans Présence de la mort (1922), prolonge Rimbaud en recreusant notamment les aspects sociaux de ces énigmes, non sans renforcer leurs couleurs apocalyptiques. (La valeur autocritique de ces énigmes revit aussi bien dans ce roman, lui-même exemplaire de penchants autocritiques, plus apparents chez Ramuz que chez Rimbaud.) Présence de la mort se lit comme un reflet anticipé du monde qui est le nôtre, en proie à des catastrophes climatiques, sociales et politiques, annoncées dans le roman par une Parole (journalistique?) dont le contenu ne cerne rien d'autre que la fameuse contradiction : un accident « survenu dans le système de gravitation » (Ramuz, $2005: 3$ ) qui fait retomber la terre vers le soleil. Avant d'évoquer les troubles sociaux engendrés par cet accident, je dois citer les idées de Ramuz, dans ses recueils de pensées, concernant "l'homme standard ", résultant d'un " programme » qui vise à "supprimer les différences » entre les hommes. Pour Ramuz, la mission du poète est d'ailleurs de «faire que les hommes ne soient plus posés les uns à côté des autres »(Ramuz, 1968:42, 58), tels des unités « interchangeables, donc déplaçables en tous sens» (Ramuz, $1968: 68$ ). Cette collection d'unités s'oppose dans l'esprit de Ramuz à l'Unité purement spirituelle que redécouvrent ses jeunes porte-paroles dans d'autres romans, et dont le sens même, dans maints passages de Présence de la mort, s'altère dans celle de la totalité violente.

12 Au chapitre 5, les explosions qui font sauter les grands rochers, « éventrant chaque jour un peu plus la montagne » (Ramuz, 2005 : 15), expriment l'acharnement contre l'unité que symbolise la montagne savoyarde, au profit du même, justement figuré dans ce passage par « les grands blocs qui dégringolent (...) comme un troupeau de moutons. »

13 Au chapitre 12, l'évocation d'un revirement sentimental révèle l'objet du symbolisme de l'accident cosmique, qui concerne un aspect très répandu de la cyclothymie des hommes, laquelle exclue toute aspiration à l'unité : «On sera pris séparément, un et un. (...) Unité, où es-tu, de nouveau? je suis deux.» (Ramuz, 2005 : 32, 33). A l'exception du dernier chapitre, le roman est semé de détails illustrant la dévalorisation des êtres, tous identiques, fondus dans un ensemble qui est celui de la totalité la plus violente. « On sera pris séparément, un et un », observe l'amoureux déçu du chapitre 12 ; autrement dit nous serons des individus indépendants et solitaires, au lieu de ne faire qu'un.

Cette débâcle sentimentale évoque d'ailleurs le nouveau visage de la mort, cerné au chapitre 4 par un quidam résigné à la catastrophe attendue: "On avait l'habitude de mourir chacun pour son compte (...). Il paraît que ça va changer... Tous ensemble !... Toi... toi.... moi... et puis eux... » (Ramuz, 2005 : 12). Cette uniformité, loin de réfléchir l'Unité du Verbe, accompagne une dégradation du sacré, lui-même chosifié parmi les réalités du monde, devenues équivalentes. Ainsi les églises, au chapitre 6, sont sur le même plan que les tramways : " on apercevait [...] le même grand pont à arches de pierres, les mêmes toits, les mêmes passants, les mêmes tramways, la même haute tour de la cathédrale à un même endroit dans le ciel. » (Ramuz, 2005 : 16). Mais c'est au chapitre 15 (au beau milieu du roman) que se dramatise avec tout son éclat la violence inhérente à l'abolition des « différences ».

L'accident déjà souligné ne symbolise rien d'autre que l' "empêchement ", ressenti au chapitre 15 par les consommateurs d'un café, dont les pulsions violentes, avivées par la permissivité absolue de ce monde en crise, se canalisent sur l'aubergiste qu'ils massacrent. Ramuz devance ici les idées de René Girard sur l'abolition des différences qui sont le ferment de la violence mimétique. Les agresseurs agissent "tous ensemble ", « s'aidant les uns les autres (...) n'étant qu'un seul (...) étant plusieurs, n'étant qu'une seule personne » (Ramuz, $2005:$ 41-44). 
16 Au chapitre suivant, ce drame s'intériorise dans l'aveuglement physique d'une famille lors d'une panne d'électricité : comme si l'indistinction des limites entre les êtres était symbolisée dans cette situation. Au chapitre 18, l'ébat des danseurs et des chanteurs n'annonce rien de bon: «égalité et communauté!» (Ramuz, 2005: 54). En effet, l'un d'eux monte sur un banc, prenant sa partenaire "à bras-le-corps » ", pour «être plus haut qu'eux (...) au-dessus des corps, au dessus des têtes (...)/ Puis : pan! un seul coup de fusil. / Le garçon et la fille dégringolèrent ensemble. » Cet « ensemble » ne manque pas de sel ; loin de s'opposer aux mentions négatives de ce mot, il en exprime les enjeux sacrificiels et violents. Et le narrateur de justifier : " parce que tous ensemble, tous à la même hauteur, n'est-ce pas? et égalité. Il n'avait pas le droit, c'est bien fait. » Ce respect forcené de l'égalité est le revers d'un désir mimétique impossible à satisfaire : celui de la foule envers ces jeunes gens, mais d'abord leur désir de hauteur, non moins pervers?

17 Ce chapitre 18 nous vaut d'ailleurs le point culminant du thème du faux-semblant, notamment avec «ce faux ciel sur la terre [qui] nous pèse dessus [...] mais c'est tant mieux ! (Ramuz, 2005 : 53). Il s'agit sans doute des fumées des maisons incendiées, dans la suite de ce chapitre. Or, ce « faux ciel » est le signe de la dévalorisation de chaque être produite par l'égalitarisme; l'être de chacun se vidant à ses propres yeux comme à ceux d'autrui, au point que sa réalité devient incertaine, aléatoire. Ce climat influe sur les goûts les plus répandus des hommes, qui avantagent toutes les formes du faux-semblant que leur impose l'industrie moderne. Le texte de Ramuz, dans ce chapitre même, ne manque pas de le signifier, avec la «mécanique (...) obstinée » de l'accordéon et, mieux encore, le « faux marbre » (Ramuz, $2005: 54$ ) des grandes banques soumises au pillage, vainement substituées aux temples, avec leurs «colonnes ». Le monde animal n'est pas moins parlant, avec le sort des vaches en souffrance qui «s'imitent toutes " (Ramuz, 2005 : 73) au chapitre 25. Cette leçon prolonge d'ailleurs celle de l'auteur de Une saison en enfer, qui fustige les illusions de l'art en se traitant lui-même de "saltimbanque ». Chez les deux poètes, la critique du faux-semblant accompagne celle, plus subtile, des formes textuelles régies par la symétrie. Dans ces passages, l'accordéon lui-même, comme les colonnes de la banque, sont autant d'images dérisoires de ce phénomène. De même avec l'allégorie plus voyante et mieux connue de la vannerie, dans d'autres récits de Ramuz.

Dans un long passage du chapitre 29, la métaphore spéculaire des eaux « lisses comme du métal », dans lesquelles finit par tomber un avion, après une ascension suicidaire dans un ciel trop chaud, exprime elliptiquement le rapport du mimétisme et de la contradiction, dramatisée dans maints détails du vol de l'avion. Cette métaphore se rapproche du «miroir» incandescent de Mémoire de Rimbaud; et comme lui, se lit comme un signe d'une remise en cause des procédés mêmes de l'art poétique, que Ramuz n'a pas empruntés à Rimbaud. Dans ce passage, la vision des hommes « agenouillés devant leurs dieux visibles ou invisibles [...] les ayant maudits, les ayant priés » » (Ramuz, $2005: 50,75)$, réintroduit le sacré dans cette énigme. Les dieux sont d'ailleurs remplacés, vers la fin du roman, par les préfigurations des prouesses technologiques de notre époque: le "sépulcre mécanique" d'un "pendule régulateur", ou bien les "grandes turbines électriques ", assez vite suivies par le constat de la destruction à laquelle n'échappent pas "les points cardinaux eux-mêmes » (Ramuz, $2005: 88,89$ ). Le symbolisme de ces détails intéresse la cyclothymie des hommes, à laquelle ces métaphores industrielles ôtent même son humanité. 
Personnage fugitif du roman, Gavillet, un comptable, occupation allégorique entre toutes, apparait au chapitre 6 et, dans une symétrie attendue, au chapitre 23, où il prend une résolution mortelle : «Voilà comment l'homme est fait, ce rien qui est tout, puis il n'est plus rien du tout. Gavillet voit qu'il va ne plus rien être, et il a tellement peur de ne plus être qu'il pense : 'Plutôt n'être plus !' Voilà comment les hommes sont faits. Ils vont à la mort par peur de la mort [...] ils sont attirés par le vide même» (Ramuz, 2005: 68). Loin d'être seulement pascalienne, cette vision trouve sa raison dans la contradiction intime, si proche de celle que Ramuz pressentait au fond de son être, mais que ce comptable suicidaire, confronté à son miroir, ne peut résoudre dans l'art : « Il se met à se haïr, puis il s'aime (...) repoussé (...) attiré (...) il se fuit, il se court après. » L'évocation des banques n'est pas moins parlante: "espèces de forteresses qui provoquaient l'envie en même temps qu'elles l'interdisaient » (Ramuz, $2005:$ 55, 67).

La détonation du chapitre 5 trouve un écho parfaitement symétrique au chapitre 28 , avec les « espèces d'explosions, comme quand on fait sauter un tronc d'arbre » (Ramuz, 2005 : 85). Ce passage est d'ailleurs rempli de symboles de l'harmonie rompue, une harmonie que Ramuz maîtrise dans la construction de son roman, suivant son credo, énoncé dans ses Remarques: "Toute vraie composition est concentrique. [...] Toute composition véritable a un système de gravitation.» (Ramuz, 1987:22). Ce système trouve une expression métaphorique dans le «système de gravitation » accidenté de la terre, évoqué au début de Présence de la mort. On comprend mieux la nature et les moyens de la mission du poète, telle que Ramuz l'envisage dans d'autres essais.

21 Cette mission a été aussi un peu celle de Henri Bosco, autre poète et romancier métaphysicien, qui a souvent exposé sa théorie de la littérature en recourant à des symboles cosmogoniques ou religieux. Sa réflexion sur la catharsis poétique, implique le rapport mystérieux de la forme textuelle et des pratiques sacrificielles, thème récurrent de ses récits. S'il se rapproche de Ramuz pour avoir été injustement considéré comme un écrivain du terroir, Bosco a été plus discret sur sa dépendance à l'égard de Rimbaud, malgré les nombreuses allusions, ironiques certes, à ce dernier dans son premier roman publié, Pierre Lampédouze (1937). Ce roman est surtout exemplaire de l'acuité du regard que Bosco pose sur le mimétisme interhumain, tourné en dérisions dans maintes situations. Ce désir mimétique avant la lettre semble inspirer la manière dont Bosco définit la source de son imagination : un conflit entre chien et loup (impliquant un chien réel, aux apparences de loup blanc (Ytier, $1996: 173,164)$ et plus généralement celui de deux personnages dont l'amour ou la haine sont autant de conflits.

Le sens mythique de ces conflits transparait dans les récits de Bosco. Or, dans ces derniers, le mystère de la forme textuelle est aussi bien éclairé par le mythe des frères ennemis que par celui de l'Un et du Verbe, objet de la réflexion de Bosco, qui a lui-même traduit l'Apocalypse. Malgré ce credo quasi mystique, Bosco se risque en effet à une remise en cause de l'Harmonie, rimbaldienne si l'on veut, qui prend tout son relief dans L'Epervier (1963), roman tardif, imprégné par le souvenir de Billy Budd (1891), où Melville suggère explicitement le rapport entre l'harmonie de la forme textuelle de son récit et l'ambiguïté du rapport des actants, surtout le désir mimétique impossible à assouvir du lieutenant calomniateur pour le beau Billy, qui est lui-même une incarnation de la « symmetry of form» (Arouimi, 2016).

Le narrateur de L'Epervier observe les habitants du village :

Sur la terrasse, il y avait une douzaine de personnes, nombre insolite, à ce que je pensais. Et ces gens-là, insolitement aussi, s'étaient divisés en deux groupes. A ma 
droite, et devant un guéridon de marbre (moins étincelant que le mien) six formaient une société particulière, alors qu'à ma gauche, six composaient un cercle, devant un autre guéridon de marbre, et semblaient, comme les premiers, avoir quelque motif d'être assis ensemble. Entre les deux groupes, un espace vide [...] étendait une zone de séparation (Bosco, 1963 : 153). semble illustrer la position même de cet épisode où l'action romanesque est pour ainsi dire suspendue, exactement au milieu de l'espace textuel du roman. La confrontation des deux groupes représente alors les tensions qui, dans la psyché du créateur, se dépassent dans une « harmonie de forme » qui resterait à étudier. Or, quelques lignes plus loin, se lisent les phrases :

Mais le danger flottait dans l'air... / L'équilibre qui maintenait, Dieu sait par quels fils! les deux groupes (...) dans l'attente, et comme à mi-hauteur entre le silence et les voix, me semblait si fragile que j'en redoutais la rupture, qui eût précipité avec violence l'un contre l'autre les deux camps. (...) La parole reprit ses droits dans chaque groupe, mais (...) [aucun] mot ne fila intempestivement, telle une flèche empoisonnée, du groupe de la droite au groupe de la gauche (Bosco, 1963 :154).

(a férences à la « parole » confirment le sens poétique de cette énigme qui concerne le rôle thérapeutique de la «forme » et des principes qu'elle médiatise, manifestés par le nombre des actants, un nombre dont les allures cabalistiques se précisent dans d'autres romans de Bosco. d'Hyacinthe (1946), s'inscrit exactement au milieu de la troisième partie de ce roman. L'étude reste à faire de la cohérence interne de ce roman (chacune de ses cinq parties titrées se présente comme une succession de développements séparés par le signe de l'étoile). Le narrateur héberge une mystérieuse enfant, dont l'aura surnaturelle est une force contraignante à laquelle il sacrifie sa quiétude, pour un bénéfice incertain. Les deux personnages incarnent en fait l'attente de l'inspiration, et son mystère absolu. Or, le narrateur et Hyacinthe ne sont jamais mieux unis que dans leur identification implicite au renard qui « dévastait notre basse-cour »:

La peau séchait. On l'avait suspendue par l'arrière-train. Les deux pattes écartelées étaient attachées à la corde par des ficelles. (...) On avait écorché la bête brutalement. (...). Dans la gueule aux canines féroces on avait passé un bâton de coudrier. C'est une coutume du pays. Le soleil illuminait la peau sanglante qui fumait doucement. La bête séchait bien. A deux doigts des babines retroussées, une guêpe toute frémissante cherchait un point de chair où piquer son dard (Bosco, 1946 : 144).

L'unité semble retrouvée dans ce sacrifice sanglant. Le « soleil » comme le «bâton » sont peut-être les signes concurrents de cette réconciliation. Mais l'unité est aussi dans le rapport de la brutalité et de la douceur («brutalement [...] doucement»), mais encore celui du mammifère et de l'insecte armé de son dard, qui cherche « un point de chair ». Le «bâton de coudrier » ajoute un sens quasi magique à cette imagerie qui circonscrit les vertus cathartiques de la forme poétique.

L'identification de Hyacinthe et du renard est manifestée par divers détails, comme les «bras écartés » de Hyacinthe, qui l'apparentent à l'animal qu'elle découvre pendu : « elle leva la tête et vit près de sa figure le museau du renard rayé de sang. [...] Puis elle se tint immobile, les bras écartés, frappée d'étonnement et d'horreur. » On peut interpréter ces images comme une critique de l'harmonie de la forme, vue comme l'expression des 
dangereux effets de miroir où se perdent nos êtres, si le renard, double de Hyacinthe, est ici mis pour le narrateur lui-même.

L'épisode du renard se prolonge par l'évocation d'une "camomille sauvage ", arrachée par Hyacinthe « avec [une] violence » qui la distrait du balancement de «la dépouille de la bête » (Bosco, 1946, 146). Bosco résume ici les pratiques sacrificielles qui sont notre quotidien, et qui déterminent notre rapport au réel. Dans le développement suivant, le silence de Hyacinthe suit ses propos très énigmatiques qui, «hors d'elle, en nous, (...) épandaient comme une lumière diffuse et (...) troublaient ma pensée » (Bosco, 1946 : 147). Cette lumière qui n'illumine pas n'est pas seulement l'expression de la fameuse contradiction. Bosco appréhende ici l'ambiguïté de notre vision de «l'Un» (pivot de sa réflexion), cet Un que nous sommes incapables de concevoir sans lui rapporter la faille autour de laquelle s'articule notre être.

Ces vertiges du double s'épurent dans L'Enfant et la rivière (1945), où ils se voient plus directement liés au sacré : la structure de ce roman, centré sur l'invention d'un monstre fictif par les deux jeunes protagonistes, juste avant de découvrir une chapelle ornée d'une Vierge de plâtre, reproduit celle de l'Apocalypse, que Bosco venait de traduire. (Ce livre biblique est centré sur l'évocation de la "bête", et sur le combat de la Vierge et du Dragon.) La relation de Pascalet et de Gatso échappe au danger du mauvais mimétisme. Mais leur entente résulte de pratiques sacrificielles, essentiellement culinaires, avec la fumée des feux allumés.

Dès le début du récit, les cheveux «barbelés d'épines» (Bosco, 1999: 26) d'un Pascalet fugueur l'apparentent au Christ. Dans ce passage, le signe de croix tracé sur le pain par un autre personnage, confirme parmi bien d'autres détails cette ressemblance. Mais à la fin du récit, les « épines » de poissons « monstrueux » vus en rêve par Pascalet, suggèrent sur le mode démoniaque le sens quasi religieux de cette symétrie de la forme, à laquelle participent une infinité de détails du récit. Cette forme est bien la catharsis des tensions inhérentes au double (associé au monstre par Girard en lisant Shakespeare). Sur le plan de l'action rapportée, les situations où revivent les anciens sacrifices sont le révélateur de cette fonction poétique. Bosco (peut-être influencé par la préface du Nègre du Narcisse de Conrad) définissait le poète comme « le lieu privilégié où se croisent [des] forces (...) ces ondes (...) communiqu[ées] par des mots» (Ytier, 1996: 19). Des mots qui ne sont que le médium de ces forces, ces tensions ressaisies dans une "alchimie du verbe " généralisée, commune à ces trois poètes, et qui ne relève pas de l'apprentissage ou de l'imitation littéraire.

\section{BIBLIOGRAPHIE}

AROUIMI, Michel (2016). Ecrire selon la rose. Paris : Hermann.

AROUIMI, Michel (2009). Vivre Rimbaud selon Ramuz et Bosco. Paris : Orizons.

Bosco, Henri (1999). L'Enfant et la rivière. Paris: Gallimard [Folio].

Bosco, Henri (1963). L'Epervier. Paris: Gallimard. 
Bosco, Henri (1946). Le Jardin d'Hyacinthe. Paris : Gallimard.

RAMUZ, Charles-Ferdinand (2005). Présence de la mort in Romans II. Paris : Gallimard [Bibliothèque de la Pléiade].

RAMUZ, Charles-Ferdinand (1987). Remarques. Lausanne : L’Âge d'Homme.

RAMUZ, Charles-Ferdinand (1968). Taille de l'homme, in Euvres Complètes 15. Lausanne : Rencontre.

RIMBAUD, Arthur (2009). Euvres Complètes. Paris : Gallimard [Bibliothèque de la Pléiade].

YTIER, Robert (1996). Henri Bosco : l'amour de la vie. Lyon : Aubanel.

\section{RÉSUMÉS}

Certains poèmes de Rimbaud mettent en scène les origines mêmes de la violence dont ces poèmes sont une sorte d'exorcisme, ou le remède. Il en va de même dans les romans de C.-F. Ramuz, désireux de « revivre » Rimbaud sans le copier. Les romans de Henri Bosco (lui-même préoccupé par Rimbaud) ne sont pas moins révélateurs : certains passages se lisent comme une synthèse des rituels sacrificiels étudiés par René Girard. Et comme chez Rimbaud, le désir créateur se voit impliqué dans cette énigme.

Some poems of Rimbaud seem to show us the deepest origin of violence, as a sort of cure or exorcism made in (or by) the poem. The same happens in some novels by C.-F. Ramuz, who wanted to « live » Rimbaud, without any imitation. Many novels of Henri Bosco (often associated with Ramuz by french critics) can be read like a synthesis of sacrificial rituals, studied by René Girard. And the act of writing is linked to this enigma.

31 janvier 2018

\section{INDEX}

Mots-clés : auto-analyse, double bind, poésie, rituel sacrificiel

Keywords : self-analysis, double bind, poetry, sacrificial ritual

\section{AUTEUR}

\section{MICHEL AROUIMI}

Université du Littoral

arou2[at]wanadoo.fr 